\title{
Analysis of Morphological Aesthetics
}

\section{Fang He}

Tianhua Advertising Co., Ltd., Jinan, Shandong, 250000, China

\begin{abstract}
Modern art design is the product of the combination of art and science and technology, it is a creative activity that injects human aesthetic ideals and aesthetic experience into human thought. Morphological aesthetics refers to the aesthetic issues involved in the creation of material culture, which is mainly reflected in the aesthetic creation of modern human technology, the core of its research is the aesthetic interpretation of the shape and nature of the products designed in the environment. Therefore, the paper studies morphological aesthetics, to enhance the aesthetic experience of the individual.
\end{abstract}

\section{Keywords}

morphological aesthetics; art; aesthetic experience

\section{浅析形态美学}

何放

天华广告有限责任公司, 中国・山东济南 250000

\section{摘 要}

现代艺术设计是艺术与科学技术相结合的产物，它是把人的审美理想和审美体验注入人的思想的创造性活动。形态美学是指 在物质文化创造中所涉及的美学问题, 主要体现在现代人类技术的审美创造中其研究的核心是对环境中设计的产品的形状和 性质的美学解读。因此, 论文对形态美学进行研究，旨在提升个人的审美体验。

\section{关键词}

形态美学; 艺术; 审美体验

\section{1 形态美学的概念}

形式美学是研究形式在视觉设计中的美学意义, 具有广 义和狭义之分。狭义的形式是指事物的外观或风格, 以及相 对于内容的形式。内容决定形式, 形式服从内容。它体现在 形态形成规律和视觉认知上, 这是一个狭险的形式概念。广 义的形式是物体空间所占有的等高关系和表面关系，可以是 平面关系，也可以是三维关系。

形式是眼睛所持物体的基本特征之一。但无论形式的自 律多么纯粹和丰富, 一旦成为设计的形式语言, 所有的形式 都应该成为某种东西的外在表达。所谓形式是与功能无关的, 因为艺术设计是不必要的。没有任何实际功能的纯粹形式不 再是设计的对象, 而是纯粹艺术的概念。形式美学研究狭义 的形式关系。

\section{2 形态的构成要素}

\section{1 形态的基本要素}

2.1 .1 点、线、面

点、线和表面是形式的基本元素。根据形式系统上经典 几何的认知方法, 认为形式的形成总是从军事维度上 “点” 的机械运动开始的。平面是一条直线，几何的经典定义，强 调了元素的机械运动对形成地层的重要性。作为 “形成” 的 概念, 点、线和面等元素是形成形式的逻辑起点。

(1) 点

点是我们实际生活中经常能体会到的，例如，当笔尖在 纸上轻轻点一下, 就形成了一个点; 当下雨时, 雨是以点的 形式落下来的, 并且落在窗户上还是会形成点状; 当我们和 人道别, 远远看去他消失的背影, 最终形成一个点, 然后才 会消失在我们眼前。点无处不在，也存在于日常的形状中， 
例如, 线段的两端就是点, 在多边形线的转折处、三角形的 顶点、圆锥的顶角都有点的存在。点在立体造型上的特点是 确定位置。它在造型学上的特性是通过凝聚视线而产生心理 张力。

在 3D 体系结构中，点是表示空间位置的视觉单位。不 论其大小、厚度和形状如何, 只要与周围的其他形式相比具 有视线集中和空间位置的特征, 它就是我们可以称为 “点” 的最小视觉单元。换句话说，点的概念不是绝对的，因为在 三维构成中, 没有几何意义上的实点, 只有相对比较。当你 和蚂蚁在一起时, 如果你是一个 “身体” , 这是把你比作一 座建筑的关键。因为点在视觉体验上有一个浓缩的视线, “点” 的形状可以很容易地引导我们的视觉去关注它, 如晚上的灯 塔、暗房里的灯光、晚上的夢火虫、衣服上漂亮的纽扣等, 都会引起我们的注意。如果在视野中存在两个性质相同的点, 那么我们的视线就会在这两个点之间来回移动, 从而形成一 条在表面不可见的线。如果有三个点来回, 就会有一个假想 的三角形, 如果有无数个具有相同属性的点, 它将形成一个 虚拟的面孔, 如夜空中的银河系。由于点的大小、亮度和距 离不同, 点的组成可能会不同, 造成不同的效果。

大小、亮度、距离相同的小圆点给人一种光滑、有序、 均匀的感觉, 但明显单调。不同大小和距离的点可以产生三 维效果。不同亮度的点可以重叠, 产生丰富的层次感和立体感。 虽然点是建模中最小的视觉单元, 但由于集中的视觉特征, 其往往成为影响整体建模的重要因素。

\section{(2) 线}

电缆、栏杆、头发、树枝和筷子给我们一种真正的线条感。 线既有直观的线，也有非直观的线，如单面线。一条线沿着 特定的路径移动, 形成一个曲面。线在造型学上的特点是表 达长度和轮廓。在三维构图中, 虽然没有几何线条, 但只要 厚度限制在必要的范围内, 与周围其他视觉元素相比, 连续 的特征、表达长度和轮廓特征都可以得到充分的表达。两者 都可以称为线。根据它的存在, 它可以分为积极的和消极的 类型。所谓的正线，是指一条独立存在的线，以及一条以导 线或绳索等三维形式存在的线。“负线” 是指具有平面或三 维边缘的非相依线。线条，因为粗、细、直、滑的程度不同， 给人不同的心理感受。粗线给人一种强烈的感觉, 细线给人 一种软弱的感觉; 直线给人一种整体的力量感, 而曲线给人
一种流动和柔软的感觉。光滑的线条给人一种精致、柔和的 感觉，而粗的线条给人一种粗粘、质朴的感觉。因此，不同 的线条对三维形状的整体影响是不同的。形成一条线的方法 有很多种, 有连通的，也有不连通的，有重叠的，也有交叉的。 根据线路的特点，确定线路的厚度、优势、角度、方向、间距、 距离等。

( 3 ) 面

迈耶在谈到 “脸” 时说, 尽管线条的特征大多是理性的, 但二维平面却充满情感, 充满了幻想和活力。艺术家或设计 师可以在由线条围绕的平面空间中创造创意，就像地理意义 上的平面一样，也就是人们可以生活、娱乐和工作的空间。 表面的产生是 “线” 的运动轨迹。由于外力的影响, 线有不 同的运动，从而产生不同形状的表面。面孔可以形成不同类 型的形状，如简单形状的面孔、复杂形状的面孔、平面形状 的面孔、三维空间的表面。康定斯基称构成作品的平面空间， 即基础, 称为 “基本平面”。他认为, 这是 “包含作品内容 的基本空间，它由两条水平线和两条垂直线形成，与周围空 间分隔并隔离开来, 并具有独立存在的性质”。基本平面在 没有内容且独立存在的情况下具有表现力, 这是由意识对重 力的不同分布引起的感觉。当心理学家研究这个问题时, 他 们认为人们倾向于认为左侧的结构较薄而右侧的结构较重。 此功能可能与一个人的左右手习惯和大脑控制有关。

\section{1 .2 体}

实际上, 任何形态都是一个 “体”。在建模中, 体有三 种基本形状，即球体、立方体和圆锥体。按形成形式可分为 半固态、点固态、线固态、平面固态和块状固态。半立体以 平面为基础，局部空间以立体为浮雕；点立体是点状的空间 视觉连接形式，如灯泡、气球、珠子等; 线立体是以线的形 式生成物体的空间。长度，如铁丝和竹筏，是由空间中的平 面形式 (如镜子、书籍等) 形成的重量和体积的三维形式, 在空间中形成一个完全封闭的实体，如石头、建筑物等。

\subsection{3 形状}

形状是眼睛抓住的物体的基本特征之一。它是指不包括 对象的特殊特征（例如, 其在空间中的位置和方向）的外部图 像, 即, 形状与对象放置的状态无关, 而仅与对象本身和对 象的边界有关。眼睛和物体之间的关系: 形状是物体在距离、 角度、光线和空气环境的特殊条件下的外观。对于同一物体, 
不同的视角将导致不同的形状。例如，立方体可以呈现正方 形或正六边形, 这恰好是由眼睛看到的对象的不同边界轮廓 引起的。形状是形式的基础, 并且是特定条件 (主要是视角) 下物体的单个表面。客观的三维空间始终由多个相同或不同 的单面组成。人脸与人脸之间的关系是形式, 并且这些关系 的总和构成了该物体的整个图像。因此, 形状、形式和图像 是相互关联的、不同的概念。

\section{1 .4 色彩}

色彩是人眼睛的对应物, 也是造型的重要元素。色彩是 物质表面的物理属性对可见光线吸收和反射后形成的不同波 长的光线作用于人的视觉感官后所产生的特性。色彩对人视 觉的影响力虽然不如形状持久, 但却是重大而强烈的, 它在 设计的形式构成中具有特殊的视觉作用和形象作用, 因为不 同的色彩会产生不同的视觉感受，表达不同的意义。

色彩有固有色、光源色和环境色之分。光源色的状态、 物体受光的方式和环境都会改变视觉对色彩的感受性。尽管 色彩与形状是不可分的, 但是色彩仍然是一个相对独立的系 统，它包括色彩的物理、生理和心理等诸多方面的特性。

\subsection{5 肌理}

肌理是物体表面的纹理。不同材料的表面通常呈现不同 的肌理。因此, 肌理通常可以反映物体的物理特征, 如木材、 岩石、金属、玻璃、水等。肌理经常给人不同的视觉感受， 如光滑粗鋉、坚硬柔软、干湿透明和不透明。肌理一般可以 分为以下几种: 自然肌理, 属于自然肌理的性质。模仿肌理, 就是用一定的工具和方法模仿自然肌理。人工肌理，是指由 一些工具和方法创建的肌理, 包括人造产品的肌理。视觉肌理, 是眼睛可以直接看到的肌理。触觉肌理, 是一种非平面肌理, 可以通过触摸感知。肌理不仅能增强物体的物理属性, 还能 增强视觉空间感。例如, 如果对象出现在平面上, 肌理可以 增强其空间的深度和分量。三维物体也是如此。肌理清晰的 物体总是首先吸引人的目光。肌理也有很强的装饰性, 可以 丰富眼睛的视觉体验。

\section{2 形态的关联性要素}

\section{2 .1 数}

“数” 是一种视觉力, 形态与数的关联极为密切, 形态 的表现实际是一种数的表现, 这是由数的 “量” 的变化而构 成的一种空间的变化。在设计形态中 “数” 的概念有不同的
表现形式。

(1) 逻辑的数

形态构成中的 “数” , 很多时候表现为一种空间、形态 关系的逻辑性结构。最典型的例证就是黄金矩形，长与宽的 比例尺度就是一个数的逻辑性结构, 即这个数是人类优化出 来的, 这个关系概念是一种数列关系, 构成设计中还有许多 通过数列发展起来的空间关系，如费波那契奇数列：第三个 数是其前两个数的和 $(1,2,3,5,8,13, \cdots)$, 这个数 列可以将空间或形态分解成复杂而又充满秩序的结构状态。

\section{(2) 重复的数}

重复的数不像逻辑的数那样仅仅是一种关系, 而是一个 直观的可以见到的数。重复的数把相同或类似的形态不断地 重复以形成量, 并通过这个量去产生视觉的影响力。很多形 在单个的时候没有什么力量, 然而当它不断扩张重复时其增 加出来的量, 就可以吸引住眼睛。“重复就是力量” , 这个 力量就是由 “数” 带来的。因此, 重复在构成设计中是最基 本的形式手段, 也是最需要理解和加以重视的概念。

\section{(3) 对称的数}

形态中的数的变化, 最简单的方式就是自我复制, 当第 一次复制完成时，形态的关系就是一个对称的关系。空间形 态中数的对称是一个数加人一个相同的数, 简单的方法就是 利用镜子，把一面镜子放在一个形面前，立即会复制出一个 镜像来, 它们之间的关系就是一个镜像对称的关系。有趣的是, 这个对称的关系是由虚与实的两个形态共同构成的。

\section{(4) 分解的数}

形态中数的增殖常常依靠自身分解的方法, 由 1 变成 2 , 再变成 4 , 如同细胞的生成。分解的数使形态具有 “分形” 的 特征, 形态不断地迭代构造或是自我仿射而形成一种“自相似” 关系。这种分解的方式能够将空间或形态的样式构筑成一个 既无穷变化, 又保留了母体特性的构成关系。这种分解的方 式也是一种数列的关系, 即在一个单纯的几何形空间中找到 个数的关系, 然后根据这个数分解出与前一个形相同的形出 来, 然后再依次分解 ${ }^{[1]}$ 。

\subsection{2 变化}

视觉模式的丰富性和设计模式的多样性往往是由变化引 起的。变化的作用之一是减少重复的单调, 带来新的视觉感受。 变化可以说是设计的武器, 它可以不断发展视觉风格, 获得 
新的视觉体验。从形态构成上看, 变化是一种力, 而形态和 空间的变化是由力的变化引起的。首先是消极运动的形态。 由于力的变化, 它可以逐渐转变成一个正向运动的形状。因此, 变化的形态往往直接反映了这种力的运动特征。设计模式中 的许多运动模式都是由变化形成的 ${ }^{[2]}$ 。

\section{2 .3 编排}

排列对形态的影响是积极的。它是形态组织、组成和管 理过程中形态关系的构建。从某种角度讲, 编排可以说是一 种技术上的组合手段, 在形式组合中起着重要的作用。编排 的核心功能是组合不同的元素并构建有序且可读的空间序列。 它的有序性是由形式编排创建的可读的视觉过程。因此, 编 排本身就是一种表达形式。不同的编舞和不同的视觉过程将 传达不同的含义。因此, 编排并不是纯粹的技术, 它还需要 创造力、构想和独创性。图形设计中的图形布局是很多单个 屏幕, 包括文本、符号、标题和其他元素集成在一起。布局 所涉及的元素比单个图片的组成要复杂得多, 因为布局设计 的空间形式不仅是单个独立的平面空间, 而且还是由多个书 页组成的序列化空间。涉及的要素包括信息、空间和时间。 布局的概念也存在于三维空间的设计中, 其形式主要出现在 展示空间或环境空间中。例如, 环境设计和展示设计中的布 局规划布局是一种广义的布局样式。城市规划是最大的空间 布局之一。

\section{2 .4 构成}

构成具有结构、形成和形状的意义。构图通常关注元素, 关注点、线、面、基本形状、骨骼、方向、力、颜色、纹理、 位置等的性质和功能。然而, 对这些元素的理性分析并不是 最终的目标, 最终的目标是最有效地发挥出这些元素的作用, 建立元素之间的关系, 形成独特的风格。作品的构成更多的 是关于作品中的理性元素。例如, 重复组合和渐进组合分别 基于不同的理性逻辑和机械结构。构图也是一个技术概念, 就是通过具体的构图分析来研究建筑造型的方法和技巧, 探 索如何通过重复、分级、近似、展开、积累、分解来形成设 计构图的能力。组合与排列之间有许多共同的元素, 甚至这 两个概念也可以多次互换使用, 因为在排列中组合元素太多, 也包含了许多排列元素。但在构图上更注重相对独立的空间 风格, 造型和结构构件更加集中。我们可以使用点、线、面、 形状和颜色来创建空间样式, 但通常我们不使用它们来安排
空间样式 ${ }^{[3]}$ 。

\section{2 .5 意象}

所谓的形象就是形式的表现特征。图像是形式形成的重 要目的。如果表单缺少图像或无法形成适当的图像, 则从某 种角度来看毫无意义。实际上, 没有形式艺术是为了形式。 即使是纯形式的组成也具有很强的表现力, 并且可以形成 图像。

表单形成的图像来自表单样式的可读性。所谓的可读性 是由形式中的元素和关系所呈现的清晰可辨的状态。这种可 识别性并不意味着是否可以清楚地看到图片和空间中的形式, 而是意味着是否可以感知形式上的关系。这种感知是直观的, 不需要理性的思考以及分析和判断。

形象, 也可以说是 “机械构造的思想” 。在比较机械 “精 神” 的同构性之后, 图像以表演的形式表现出来, 而人们以 精神活动的形式表现出来, 是神经系统中唤醒人脑的运动特 征的结构形式。

意象结构是指形式构成中的隐性表现力。这种表达形式 由视觉形式中的 “运动” 和 “力学” 的结构属性组成。当视 觉观察直接掌握这些结构的性质时, 在人脑的神经系统中, 同样的力量被唤起, 并且 “紧张” 或 “移动” 的倾向被传递, 从而感知形式风格的表达, 这是象征心理学形式的基础。图 像表达是人类感知的优先事项, 因为它是我们日常视觉活动 的主要内容。像 “塔” 和 “桥” 这样的形式本身表现出与其 功能相匹配的正式结构。“塔” 的崛起动力是满足精神升华 和与天地沟通的精神形象; 这座桥是一样的。这座桥本身不 是连接道路的桥梁。它是一种空间形式, 但作为桥梁的空间 节点, 它可以作为两个封闭边界的通信节点和通道 ${ }^{[4]}$ 。

研究形式构成要素的目的是理解这些要素与设计形式之 间的关系。要理解形式构成了作为形式的内容, 同时服务于 它自己的自律。一方面, 它是形式本身的象征, 象征意义和 意象的表达; 另一方面, 这些在很多情况下是视觉艺术的灵魂。

\section{3 形态的分类}

形态具有多种分类方法, 可以依据形态的要素、结构、 关系、意义、表现和形成的方法等进行不同的分类，此处列 举几种最基本的形态分类。 


\section{1 几何形}

几何是一种简单、简洁、合理的形式，是形式世界中最 基本的形式。常见的圆、正方形、三角形等是最基本的形式。 几何学通常是高度规范的, 隐藏着严格的逻辑尺度和复杂的 数学关系。而真正的标准几何在自然界是罕见的。几何是数 学推导的结果, 通常需要特定的映射工具来绘制。因此, 几 何图形是可以完全重复和任意复制的图形 ${ }^{\left[{ }^{[}\right]}$。

几何学有时是无机物的一种形式, 也被称为 “机械形状”。 其机械严谨的结构和简单的直线、弧线和方向感往往显示出 美丽的力量和速度。因此, 几何也更加刚硬和冷漠, 是一种“冷” 的形式。但是几何学经常能揭示宇宙本质的结构关系。

\section{2 有机形}

有机形式基于自然形式。所谓 “有机” 是指具有自然生 命的有机体, 是生命的外在形式。此外, 一些无生命的物体, 如海滩上的鹅卵石和许多人工流线物体, 主要是由自由曲线 在生命运动和自然力学的作用下形成的, 可以是有机形式。 因此，它通常被称为 “生命的抽象”。

一般来说, 有机形式的形成不依赖于数学或机械方法, 而是依赖于相对自由的条件、平稳的运动。有机形式是内在 张力和外在力量相互抵抗时的和谐关系, 给人一种整体而柔 和的美感。有机形式有很强的亲和力, 是一种 “温暖” 的形式。 有机形式具有强烈的精神元素, 往往可以成为人们深层意识 的象征和暗示, 如细胞和生活的初始状态。自然界中的动物 和植物都是有机的，因此，人工作品中由曲线形成的物体往 往具有有机形态的形态特征。如果说 “流线型设计” 在当时 曾经流行，那就是一种以有机形式为主体的设计风格。

\section{3 偶然形}

这是基于形式形成的分类。偶然形式与有机形式或几何 形式完全不同，它是在意外情况下产生的不可预测的形式。 它通常是偶然的、无意的并且经常是无法控制的形式。因此, 意外形状具有偶然性、随机性和表面复杂性。墨水痕迹、裂㖓、 斑点等都是在特定的偶然情况下产生的。意外形状的复杂性 和丰富性通常会影响视觉纹理。因此, 在设计和装饰设计中,
意外形状的形成常常成为产生纹理的基本方法之一。这些方 法包括吸附、印刷、喷涂、染色等。现代计算机图形处理软 件具有许多过滤功能，可以模拟意外形状的变化。这是计算 机随机计算的结果，但仍有一定程度的机会，这种情况就像 彩票机中的随机数排列 ${ }^{\left[{ }^{[}\right]}$。

\section{4 自在形}

自在形又称 “自由形” 或 “任意形” , 它是一种人工形式, 由人们随意放置。所谓 “自由” , 就是一个人在没有任何压力 的情况下自然流露出来的，它是无意识的，它本身往往可以 揭示一个人的性格和情感倾向，因为它本身就是一种自然的 启示。严格地说，形式上的笔迹虽然可以重复，但其已经是 一个人的自然行为, 因此, 心理学家可以从一个人的笔迹来 判断他的心理和个性特征。

\section{4 结语}

美的形态学是美学理论体系中最具体、也是极其重要的 一环。正如没有美的事实，任何美的本质研究都是虚幻的一 样, 没有美的形态学这一环, 任何美学理论都是空洞的。因此, 对美的形态作专门的研究，无疑是十分必要的。

\section{参考文献}

[1] 盛子潮. 诗歌形态美学 [M]. 厦门: 厦门大学出版社, 1987.

[2] 徐刚. 美学意识形态: 伊格尔顿的意识形态美学批判——兼谈 “日 常生活审美化”问题 [J]. 理论学刊, 2006(02):116-118.

[3] 孟昭宇, 张宁宁. 产品设计的形态审美特征 [J]. 美术大观, 2012(01):111.

[4] 史蒂文·文图瑞诺, 杨莉莉. 淹没《马丁·伊登》的意识形态美学 [J] 国外文学, 1997(02):19-27.

[5] 张丽芬. 重新阐释马克思——特里·伊格尔顿的意识形态美学观述 评 [J]. 马克思主义美学研究 ,2003(09):294-306.

[6] 周爱民,苏建宁, 阎树田, 等. 产品形态审美综合评价的非线性信 息动力学模型 [J]. 机械工程学报, 2018(15):150-159. 